\title{
COVID-19 and regional differences in the timeliness of hip- fracture surgery: an interrupted time-series analysis
}

\author{
Davide Golinelli ${ }^{\text {Corresp., }}{ }^{1}$, Jacopo Lenzi ${ }^{1}$, Emanuele Adorno ${ }^{1}$, Maria Michela Gianino ${ }^{2}$, Maria Pia Fantini ${ }^{1}$ \\ ${ }^{1}$ Department of Biomedical and Neuromotor Sciences, University of Bologna, Bologna, Italy \\ 2 Department of Public Health Sciences and Pediatrics, University of Turin, Turin, Italy \\ Corresponding Author: Davide Golinelli \\ Email address: davide.golinelli@unibo.it
}

Background. It is of great importance to examine the impact of the healthcare reorganization adopted to confront the COVID-19 pandemic on the quality of care provided to non-COVID-19 patients. The aim of this study is to assess the impact of the COVID-19 national lockdown (March 9, 2020) on the quality of care provided to patients with hip fracture (HF) in Piedmont and Emilia-Romagna, 2 large regions of northern Italy severely hit by the pandemic.

Methods. We calculated the percentage of HF patients undergoing surgery within 2 days of hospital admission. An interrupted time-series analysis was performed on weekly data from December 11, 2019 to June 9,2020 ( $\approx 6$ months), interrupting the series in the $2^{\text {nd }}$ week of March. The same data observed the year before were included as a control time series with no "intervention" (lockdown) in the middle of the observation period.Results. Before the lockdown, 2-day surgery was $69.9 \%$ in Piedmont and $79.2 \%$ in Emilia-Romagna; after the lockdown, these proportions were equal to $69.8 \%(-0.1 \%)$ and $69.3 \%$ $(-9.9 \%)$, respectively. While Piedmont did not experience any drop in the amount of surgery, EmiliaRomagna exhibited a significant decline at a weekly rate of $-1.29 \%(95 \% \mathrm{Cl}=-1.71$ to -0.88$)$. Divergent trend patterns in the 2 study regions reflect local differences in pandemic timing as well as in healthcare services capacity, management, and emergency preparedness. 


\section{COVID-19 and regional differences in the timeliness of hip-fracture surgery: an}

2 interrupted time-series analysis

4

5 Davide Golinelli ${ }^{1}$, Jacopo Lenzi ${ }^{1}$, Emanuele Adorno ${ }^{1}$, Maria Michela Gianino ${ }^{2 \dagger}$, Maria Pia

6 Fantini $^{1 \dagger}$

7

8

$9{ }^{1}$ Department of Biomedical and Neuromotor Sciences, University of Bologna, Bologna, Italy

2 Department of Public Health Sciences and Pediatrics, University of Turin, Turin, Italy

11

12

$13 \uparrow$ Professors Gianino and Fantini contributed equally to this work.

14

15

16 Corresponding Author:

17 Davide Golinelli ${ }^{1}$

18 Via San Giacomo 12, 40126 Bologna, Italy

19 Email address: davide.golinelli@unibo.it 
20 Abstract

21 Background. It is of great importance to examine the impact of the healthcare reorganization 22 adopted to confront the COVID-19 pandemic on the quality of care provided to non-COVID-19

23

24 25 26

27

\section{Introduction}

42

\section{Keywords} patients. The aim of this study is to assess the impact of the COVID-19 national lockdown (March 9, 2020) on the quality of care provided to patients with hip fracture (HF) in Piedmont and Emilia-Romagna, 2 large regions of northern Italy severely hit by the pandemic.

Methods. We calculated the percentage of HF patients undergoing surgery within 2 days of hospital admission. An interrupted time-series analysis was performed on weekly data from December 11, 2019 to June 9, 2020 ( $\approx 6$ months), interrupting the series in the $2^{\text {nd }}$ week of March. The same data observed the year before were included as a control time series with no "intervention" (lockdown) in the middle of the observation period.

Results. Before the lockdown, 2-day surgery was 69.9\% in Piedmont and 79.2\% in EmiliaRomagna; after the lockdown, these proportions were equal to $69.8 \%(-0.1 \%)$ and $69.3 \%$ (9.9\%), respectively. While Piedmont did not experience any drop in the amount of surgery, Emilia-Romagna exhibited a significant decline at a weekly rate of $-1.29 \%(95 \% \mathrm{CI}=-1.71$ to 0.88). Divergent trend patterns in the 2 study regions reflect local differences in pandemic timing as well as in healthcare services capacity, management, and emergency preparedness.

Hip Fractures, COVID-19, Frail Elderly, Osteoporosis, Interrupted Time Series Analysis, Italy

Worldwide, hip fracture (HF) represents an important public health concern that determines relevant functional impairments in the individuals who experience it, especially the elderly $[1,2]$. Due to the increasing incidence of osteoporosis, the global number of HFs will reach approximately 8.2 million in 2050 [3]. In Italy, HFs accounted for 99,103 hospitalizations, $1,122,714$ occupant days and 77,543 surgical procedures in 2018.

7 The growing burden that HF causes on healthcare systems also has to do with the intensive use of healthcare resources required by this condition $[4,5]$. HF patients represent a particularly - challenging population $[6,7]$, due to the high post-operative mortality rate caused by surgery, functional impairment, and limited mobility $[4,5,8]$. 
51

52

53

54

55

56

57

58

59

60

61

62

63

64

65

66

67

68

69

70

71

72

73

74

75

76

77

78

79

80

81

Timely surgery within 48 hours of hospital admission for HF is a well-established strategy that leads to better functional outcomes and lower mortality rates $[7,9,10]$. The UK National Institute for Health and Care Excellence (NICE) and other international guidelines indicate 48 hours as the ideal time to operate on a patient with HF. Scientific evidence points out that the earlier the surgery, the better the outcomes in terms of mortality, complications, length of hospital stay, time required for rehabilitation, and patient quality of life [11-13]. For this reason, the percentage of surgical interventions performed within 2 days of hospital admission has become one of the most used health indicators to assess the performance and quality of care. In Italy, specifically, this indicator is used by the Programma Nazionale Esiti (National Outcomes Program) to measure and monitor healthcare facilities' performance and standards of care [14].

In Europe, more than three quarters (76\%) of HF patients aged 65 and over underwent surgery within 48 hours of hospital admission in 2017. This proportion was greater than $95 \%$ in Denmark and the Netherlands, and around 40\% in Latvia and Portugal [15]. In Italy, this indicator has progressively improved in recent years, reaching a national average of around $70 \%$ in 2017 [14].

In late February of 2020, the novel coronavirus disease (COVID-19) [16, 17] started to spread aggressively around many bordering provinces of the largest and most productive regions of northern Italy: Lombardy, Emilia-Romagna, Veneto, and Piedmont. The first cluster was detected in Lombardy on February 21, 2020 - in the following days, the government adopted an increasing number of decrees to limit large social gatherings, closing schools, universities, bars, and restaurants. Following the stay-at-home decree of March 9, 2020, all non-essential business and services were closed, and the entire country was put under lockdown.

The COVID-19 outbreak had a huge impact on the Italian healthcare system: usual treatment pathways were disrupted, and hospitals were reorganized to face this challenge using the limited healthcare resources available [18]. The Italian National Health Service (Servizio Sanitario Nazionale [SSN]) struggled to maintain and enhance the surge capacity of services, goods and healthcare workers in order to preserve high standards of care both to COVID-19 and non-COVID-19 patients. To keep COVID-19 and non-COVID-19 patients separated, "Hub and Spoke" models were created [19], and COVID-19-dedicated hospitals were set up to isolate contagious patients. Especially during the national lockdown from March 9 to May 4, elective surgery was cancelled, and only trauma, oncologic and urgent surgeries were allowed [18]. 
82 As such, we believe that it is of great importance to examine the impact of the healthcare 83 reorganization adopted to confront the COVID-19 pandemic on the quality of care provided by 84 Italy's SSN for conditions of major public concern such as HF. In this study, we assessed 85 whether the imposition of the national lockdown on March 9, 2020 resulted in a shift in the 86 percentage of patients who received timely surgery for HF compared with that of the pre87 lockdown period. Separate analyses were performed on two of the regions of northern Italy most 88 hit by the spread of SARS-CoV-2: Piedmont and Emilia-Romagna, with 4.4 and 4.5 million 89 inhabitants, respectively, as of 2019 [20]. As illustrated in Supplementary Figure S1, Emilia90 Romagna and Piedmont were among the regions of Italy earliest and hardest affected by 91 COVID-19; in particular, the province of Piacenza, Emilia-Romagna, was one of the areas of 92 Italy hardest hit by the pandemic due to its proximity to the epicenter of the first outbreak in 93 Lombardy $[16,21,22]$.

94 Identifying similarities and differences in how the two regions faced this unprecedented 95 crisis can be helpful for setting health priorities and identifying entry points to enhance health96 system responsiveness [23].

97

\section{Materials \& Methods}

99 We collected the hospital discharge records (HDRs) of all patients admitted to the hospitals of 100 Piedmont and Emilia-Romagna with a principal or secondary diagnosis of hip fracture (ICD-9$101 \mathrm{CM}$ code 820). In keeping with the specification of the indicator adopted by Italy's National 102 Outcomes Program [14], HDRs were excluded from the analysis if at least one of the following 103 criteria was met:

104 - Non-urgent hospital admission;

- Daytime hospital care, known in Italy as "day hospital admission", which consists in a oneday admission to the hospital without overnight stay to perform diagnostic procedures and/or surgical, therapeutic or rehabilitative care [24];

- Transfer from other hospital;

- Age $<65$ or $>100$ years;

- Polytrauma (diagnosis-related group 484-487);

- Diagnosis or medical history of malignant tumors (principal/secondary ICD-9-CM code 140.0-208.9, 238.6, V10); 
113 - Death within 1 day of hospital admission and no surgery to repair HF;

114 - Admission to a spinal injury unit, rehabilitation hospital or long-term care facility.

115 Hospitalization rates were obtained as the number of hospital admissions for HF in the 116 resident population aged $\geq 65$ years per 100,000 inhabitants. Population data were retrieved from 117 the Italian National Institute of Statistics (http://demo.istat.it/index_e.html).

118 Timely HF surgery among the cases described above was defined as any of the following 119 procedures initiated within 2 calendar days after admission to the hospital: closed reduction of 120 fracture without internal fixation (ICD-9-CM codes 79.00, 79.05); closed reduction of fracture 121 with internal fixation $(79.10,79.15)$; open reduction of fracture without internal fixation (79.20, 122 79.25); open reduction of fracture with internal fixation (79.30, 79.35); total or partial hip 123 replacement $(81.51,81.52)$. We also investigated the percentage of cases surgically treated the 124 next day (day 1) and on the same day as hospital admission (day 0).

125 Hospital admission rates were standardized by sex and age $(<80,80-84,85-89, \geq 90$ years $)$

126 with direct standardization to Italy's 2020 elderly population. Percentages of surgery were 127 standardized by sex, age and enhanced Charlson index score $(0,1, \geq 2)$ [25], with direct 128 standardization to the overall population of HFs observed in Piedmont and Emilia-Romagna over 129 the study period.

130 For descriptive purposes, we also gathered some characteristics of the admitting hospitals; 131 more specifically, we collected hospital type/ownership, hospital location, and average annual 132 caseload of hip fractures.

\section{Statistical analysis}

135 Owing to the availability of multiple weekly observations in the pre-lockdown and post136 lockdown period, we performed an interrupted time-series analysis (ITSA), an example of quasi137 experimental design [26]. Lockdown-period data were collected from March 11, 2020, to June 9, 1382020 (13 weeks, i.e. $\approx 3$ months), while pre-lockdown data were collected from December 11, 1392019 to March 10, 2020 (13 weeks). To reduce any confounding factors, the same data observed 140 in Piedmont and Emilia-Romagna the year before, i.e. between December 11, 2018 and June 10, 1412019 (26 weeks), were included as a control time series with no intervention in the middle of the 142 observation period. 
A two-group ITSA regression model can be specified as:

144

$$
Y_{t}=\beta_{0}+\beta_{1} T_{t}+\beta_{2} X_{t}+\beta_{3} X_{t} T_{t}+\beta_{4} Z+\beta_{5} Z T_{t}+\beta_{6} Z X_{t}+\beta_{7} Z X_{t} T_{t}+\epsilon_{t}
$$

145 where $Y_{t}$ is an aggregated outcome variable measured at each time point $t, T_{t}$ is time since the 146 start of the study, $X_{t}$ is a dummy variable representing the intervention (pre $=0$, post $=1$ ), $Z$ is a 147 dummy variable to denote the cohort assignment ("treatment" or control), and $\epsilon_{t}$ is the random 148 error term. Here is the interpretation of the seven parameters that constitute the linear model:

149 - $\beta_{0}=$ intercept of the outcome variable in the control group;

150 - $\beta_{1}=$ slope of the outcome in the control group until the introduction of the intervention;

- $\beta_{2}=$ change in the level of the outcome that occurs in the period immediately following the

152 introduction of the intervention in the control group;

$153-\beta_{3}=$ difference between preintervention and postintervention slopes of the outcome in the 154 control group;

$155-\beta_{4}=$ difference in the level between "treatment" and control prior to intervention;

$156-\beta_{5}=$ difference in the slope between "treatment" and control prior to intervention;

$157-\beta_{6}=$ difference-in-differences of the change of level between "treatment" and control;

$158-\beta_{7}=$ difference-in-differences of slopes between "treatment" and control.

159 As anticipated by the definitions of $\beta_{6}$ and $\beta_{7}$, causal inference is provided using the 160 difference-in-differences approach, in which between-period changes in a 161 "treatment"/experimental cohort are compared with changes in a control cohort over a similar 162 timeframe. The two parameters $\beta_{4}$ and $\beta_{5}$ are useful to establish whether the "treatment" and 163 control series are balanced on the level and the trajectory of the outcome variable in the pre164 intervention period; if $\beta_{4}$ and $\beta_{5}$ are significantly different from 0 , conclusions drawn from $\beta_{6}$ 165 and $\beta_{7}$ are likely to be biased. A visual exemplification of ITSA is provided in Linden and 166 Adams [27].

167 We computed heteroskedasticity-robust standard errors, also known as Newey-West 168 standard errors, to make valid inference about the linear regression coefficients. According to the 169 Cumby-Huizinga test [28], there was no evidence of autocorrelation. 
170

171

172

173

174

175

176

177

178

179

180

181

182

183

184

185

186

187

188

189

190

191

192

193

194

195

196

197

198

199

200

In keeping with the specification of the indicator adopted by the Organization for Economic Co-operation and Development, a sensitivity ITSA was performed on HF surgery after excluding HDRs with a diagnosis of hip fracture in secondary position. All analyses were performed using Stata version 15 (StataCorp. 2017. Stata Statistical Software: Release 15. College Station, TX: StataCorp LP) [29]. The significance level was set at 5\%, and all tests were two-sided. Code and data used to produce the reported results are made available as Supplemental Information files.

Ethical approval to undertake this research was granted from the Comitato Etico di Area Vasta Emilia Centro (Submission Number IDECOdE-R [233/2019/0SS/AOUBo]). Access to administrative data was conducted in conformity with the Italian Privacy Code (Legislative decree 196/2003, amended by Legislative Decree 101/2018), which exempts from the obligation to seek written informed consent when using pseudonymized data that are primarily collected for healthcare management and healthcare quality evaluation and improvement. According to Articles 99-110-bis on medical, biomedical, and epidemiological research (Legislative Decree 101/2018), when investigators use data collected by healthcare systems or previous studies, consulting all the participants would represent a disproportionate effort, considering that safeguards such as key-coding (pseudonymization) are in place to protect the data.

\section{Results}

Hospital admissions for HF in Piedmont and Emilia-Romagna in the control cohort (December 11, 2018, to June 10, 2019) and in the "treatment" cohort (December 11, 2019 to June 9, 2020) are summarized in Table 1. Both regions experienced a drop in the number of hospitalizations in the 13 weeks following the imposition of the first national lockdown as compared to the previous 13 weeks, although the reduction was more pronounced in Piedmont (Piedmont: 148.8 to 121.6 $\times 100,000$; Emilia-Romagna: 152.2 to $128.7 \times 100,000$ ). We registered an increased concentration of admissions to research and university hospitals (Piedmont: 24.0\% to 31.4\%; Emilia-Romagna: $27.3 \%$ to $34.3 \%$ ), combined with a decrease in the relative number of admissions to LHT and private hospitals (Piedmont: $76.0 \%$ to $68.6 \%$; Emilia-Romagna: $72.7 \%$ to $65.7 \%$ ). A summary of patient and hospital characteristics before and after the lockdown is provided in Supplementary Table S1.

Results of the ITSA on HF hospitalization rates are presented in Table 2 and Figure 1. In Piedmont, in the second week of March 2020 a strong decrease in weekly hospital admissions for 
201 HF was observed as compared to the same week of $2019\left(\beta_{6}=[7.95-10.89]-[11.21-11.93]=-\right.$ $2022.22 \times 100,000,95 \% \mathrm{CI}=-4.88$ to 0.44 ), although this difference failed to achieve statistical 203 significance $(P$-value $=0.100)$. In Emilia-Romagna, this difference-in-differences of change of 204 level was weaker $\left(\beta_{6}=[8.43-10.56]-[10.37-10.98]=-1.52 \times 100,000,95 \% \mathrm{CI}=-3.69\right.$ to 0.65 , $205 P$-value $=0.164)$. In Piedmont, the drop in the number of hospital admissions was followed by a 206 weekly significant increase in the hospitalization rate $(+0.23 \times 100,000,95 \% \mathrm{CI}=0.03$ to 0.43 , $207 P$-value $=0.027)$, although the difference-in-differences of slopes was not significant $\left(\beta_{7}=\right.$ 208 $[0.23+0.10]-[0.06+0.06]=0.21 \times 100,000,95 \% \mathrm{CI}=-0.18$ to $0.60, P$-value $=0.291)$. Similarly, 209 in Emilia-Romagna the hospitalization rate increased weekly $(+0.22 \times 100,000,95 \% \mathrm{CI}=0.11$ to $2100.34, P$-value $<0.001)$, but the difference-in-differences of slopes was once again not significant $211\left(\beta_{7}=[0.22+0.21]-[0.20+0.17]=0.06 \times 100,000,95 \% \mathrm{CI}=-0.20\right.$ to $0.32, P$-value $\left.=0.629\right)$.

212 Percentages of timely HF surgery in Piedmont and Emilia-Romagna in the control cohort 213 (December 11, 2018 to June 10, 2019) and in the "treatment" cohort (December 11, 2019 to June 214 9, 2020) are illustrated in Figure 2. In all the study periods preceding the national lockdown, the 215 percentage of surgery initiated within 2 days after hospital admission was higher in Emilia216 Romagna than in Piedmont, while between March 11 and June 9, 2020 the standardized 217 percentages of the two regions were similar and just below 70\% (Piedmont: 69.8\%; Emilia218 Romagna: 69.3\%).

219 A visual inspection of the ITSA in Figure 3 shows that the pattern of change in weekly 220 percentages of surgical care for HF after the imposition of the national lockdown was different in 221 the two study regions. As confirmed by the regression coefficient estimates presented in Table 3, 222 Piedmont did not experience any raise or drop in the amount of surgery from the second week of 223 March 2020. In Emilia-Romagna, on the contrary, after an initial period of stable or even 224 increased timeliness of surgical care, there was a significant decline at a weekly rate of $-1.29 \%$ 225 for 2 -day surgery $(95 \% \mathrm{CI}=-1.71$ to $-0.88, P$-value $<0.001),-2.27 \%$ for 1 -day surgery $(95 \%$ $226 \mathrm{CI}=-3.01$ to $-1.54, P$-value $<0.001)$, and $-1.07 \%$ for same-day surgery $(95 \% \mathrm{CI}=-1.68$ to $2270.45, P$-value $=0.001)$. As shown in Table 3, the difference-in-differences of slopes reached 228 statistical significance for 2-day surgery $\left(\beta_{7}=[-1.29-1.28]-[-0.19-0.59]=-1.79 \%, 95 \% \mathrm{CI}=\right.$ $229-3.37$ to $-0.22, P$-value $=0.027), 1$-day surgery $\left(\beta_{7}=[-2.27-1.61]-[0.07+0.08]=-4.04 \%\right.$, 
$23095 \% \mathrm{CI}=-5.59$ to $-2.49, P$-value $<0.001)$ as well as same-day surgery $\left(\beta_{7}=[-1.07-0.59]-\right.$

$231[0.16+0.34]=-2.15 \%, 95 \% \mathrm{CI}=-3.15$ to $-1.16, P$-value $<0.001)$.

232 Results were virtually unchanged after excluding secondary diagnoses of HF

233 (Supplementary Table S2 and Figure S2).

234

235 Discussion

236 This ITSA was designed to evaluate whether the quality of care received by HF patients changed 237 after the imposition of the COVID-19 national lockdown on early March 2020. Our analysis was 238 restricted to Piedmont and Emilia-Romagna, two large regions of northern Italy severely hit by

239 the pandemic. By identifying similarities and differences in how the two study regions faced this 240 unprecedented crisis, helpful information can be provided for setting health priorities and 241 identifying entry points for health-system improvement in case of future recurrences of the 242 pandemic, in Italy as in other countries. Furthermore, to the best of our knowledge, there are no 243 published studies that adopted a quasi-experimental design to discern any changes in the quality 244 of HF care during the pandemic.

245 The most important result of our study is that the percentage of timely surgery for HF 246 remained virtually unchanged in Piedmont after the imposition of the national lockdown, while 247 the Emilia-Romagna system, although performing better than Piedmont before the pandemic, 248 worsened sharply until reaching a proportion below $70 \%$ between May and June 2020. This 249 remarkable finding requires an explanation that might be sought in the different capacity and 250 capability of the two regional healthcare systems to respond to the emergency, as well as in the 251 local timing of the epidemic onset.

252 We acknowledge that the ability to intervene in time on HFs relies both on patients' clinical 253 condition, such as comorbidities or clinical instability management (e.g. coagulation problems) 254 [30], and healthcare services' organization [30-32]. However, possible reasons for interregional 255 differences should be attributed to organizational rather than clinical aspects of care; indeed, 256 Piedmont and Emilia-Romagna have slightly different health systems. Like neighboring 257 Lombardy, Piedmont has a hospital-focused system that struggled in the first months to catch up 258 with SARS-CoV-2 infections, due to the lack of a strong primary care system, but that might 259 have ensured high standards of care for non-COVID-19 patients requiring specialized acute 
260

261

262

263

264

265

266

267

268

269

270

271

272

273

274

275

276

277

278

279

280

281

282

283

284

285

286

287

288

289

290

settings [33]. Emilia-Romagna relies on a mixed healthcare system, with strong hospital facilities and a well-developed primary care network.

The pandemic hit Emilia-Romagna harder and earlier than Piedmont. Official data report that Piedmont surpassed Emilia-Romagna's hospital burden of COVID-19 on April 10, 2020, about one month after the imposition of the national lockdown, and that reached EmiliaRomagna in terms of cumulative incidence of COVID-19 on April 22, 2020, about 6 weeks after (Supplementary Figure S1) [16, 21, 22, 34]. This may suggest that Piedmont had more time than Emilia-Romagna to organize a proper response to the crisis.

Given these considerations, for the first time since data recording began, the percentage of timely HF surgery in Emilia-Romagna dropped off. This is most likely due to organizational issues related to the emergency, such as the management of testing procedures or the enhancement of the overall capacity of the healthcare facilities. The need for COVID-19 testing procedures before being admitted to the operating room (OR) is an organizational factor that may have increased waiting times. Similarly, waiting for preoperative cardiac tests and other laboratory results may have played a role in delaying surgery [31]. A recent study describes how in Lombardy's hospitals, for instance, patients were isolated at admission and sent to a "filtering" ward until the result of nasopharyngeal swabs became available, with a mean response delay, at that time, of 12 to 24 hours [35]. However, our findings cannot be generalized to non-elderly populations and to conditions other than $\mathrm{HF}$, given that a number of treatment pathways succeeded to maintain high standards of care in Emilia-Romagna. For instance, although overall hospital admissions decreased in a way similar to those for HF, management and outcomes of patients hospitalized with acute myocardial infarction during the same period remained unchanged [36].

Another aspect to be considered is the workforce surge capacity of healthcare facilities during the pandemic. After the transfer of human resources to sustain intensive care units (ICUs), anesthesiologists may have been under pressure to divide their time between ICUs and ORs, orthopedic surgeons may have been underused or assigned to internal medicine activities in COVID-19 wards, and nurses may have had to adapt to different surgical procedures [35]. Furthermore, observing COVID-19-related safety protocols is a time-consuming activity that may have created further delays. As a matter of fact, this has an impact on ORs' capacity and readiness, increasing both the "first-case delay", which is an indicator of the delay from the 
291 scheduled time for skin incision on the first patient of the day, and the "turnover time", which is 292 the time required for the exit of the patient from the OR, room cleaning, and the entrance of the 293 next patient [35].

294 All these critical aspects might be responsible for the delay in the treatment of patients with 295 HF in Emilia-Romagna after the pandemic outbreak. This highlights the importance of pandemic 296 preparedness and response plans that should include healthcare management issues to respond

297

298

299

300

301

302

303

304

305

306

307

308

309

310

311

312

313

314

315

316

317

318

319

320 not only to patients directly affected by the pathogen but also to other patients needing healthcare assistance, such as those with HF or other acute and chronic diseases. These plans should incorporate, for instance, the implementation of specifically trained professionals' networks supported by "health crisis" guidelines to maintain high standards of care even in times of pandemic emergency. Regional healthcare systems should provide healthcare personnel with disaster medicine training courses and reckon on trained "reservists" to be called back in the case of need. Moreover, hospitals and healthcare facilities should schedule simulation trainings and stress tests to speed up peri-surgical activities to be prepared in the event of recurrent crises.

Our findings also show that HF hospital admissions declined in both regions during the first weeks of the lockdown, although the difference-in-differences of change of level was slightly more pronounced in Piedmont than in Emilia-Romagna. This coherent reduction in hospital admissions suggests that the overall impact of the pandemic was similar in the elderly HF populations of the two study regions. This decline has several possible explanations.

Due to the uncontrolled spread of the epidemic across the regions of northern Italy, the central government declared the national lockdown on March 9, 2020. Lifestyle changes, fear of the contagion and sense of civic responsibility [37] may have determined an overall reduction in the number of patients accessing emergency departments (EDs) and hospitals, as confirmed by previous research conducted in Italy and other countries [38, 39]. Moreover, by confining people at home, interrupting work activities and reducing road traffic, the frequency of travel- and workrelated injuries dropped [40]; as a consequence, ED visits and hospitalizations for trauma have decreased. Some authors report that domestic accidents had a relative increase of $94 \%$ on total accesses to trauma facilities [41], determining an increase in the proportion of hospitalizations for HF in the age group $\geq 65[42,43]$ during the first wave of the pandemic. However, in Piedmont and Emilia-Romagna, despite the continued occurrence of domestic accidents, the 
321 reduction in overall mobility due to the lockdown led to a decline in the number of HFs and 322 consequent hospitalizations.

323 Lastly, we found a relative increase in HF hospital admissions to third-level hospitals. We

324 expect this to be the consequence of the reorganization of the healthcare services of both regions.

$325 \mathrm{HF}$ and traumas activities were shifted to dedicated hubs, chosen at the regional level among 326 those with more experience and treatment capacity/volumes, in order to maintain high-

327 performance levels even under stressful situations [18, 40, 44]. These decisions were made to 328 ensure a rapid increase in the number of ICUs, allowing recruitment as well as replacement for 329 healthcare workers to better assist COVID-19 patients.

330

\section{Strengths and limitations}

332 The results of this study should be interpreted considering its strengths and limitations. ITSA is a 333 quasi-experimental research design with a potentially high degree of internal validity, and the 334 addition of a control group (i.e., 2018/19 data) strengthens the causal inference that can be drawn 335 from its results [45]. By standardizing rates, we also accounted for individual-level confounding differences to evaluate the outcomes of interest at the population level, but ITSA does not allow inferences about the patients that make up the experimental and control cohorts; moreover, our estimates might be affected by some residual confounding due to the impossibility of obtaining relevant information such as patient frailty and clinical complexity from administrative databases. Another limitation to our study is that we did not have access to the hospital reorganization protocols of Piedmont and Emilia-Romagna, so we could not test which one of several potential factors played the leading role in determining our findings. Other limitations are common to all studies based on healthcare administrative data, including lack of accuracy and 344 differences in the coding criteria over time as well as across individuals and institutions.

345 However, there is no reason to believe that such potential source of information bias might have 346 significantly affected our difference-in-differences estimates.

\section{Conclusions}

349 In this quasi-experimental study, we found that the COVID-19 pandemic had a similar impact on 350 the HF hospitalization rates of Piedmont and Emilia-Romagna, two of the regions hit earliest and hardest by the virus in Europe, with a coherent relative reduction in HF-related hospitalizations. 
352 Conversely, the healthcare services response was different: Piedmont managed to maintain pre353 pandemic standards of care, while Emilia-Romagna performed worse despite starting from a 354 better performance level.

355 Our findings show to what extent the percentage of timely surgery for HF was modified by the 356 pandemic, reflecting local differences in terms of healthcare management, emergency

357 preparedness and response factors. Although there is urgent need for timely and effective 358 management of COVID-19 patients, it is essential not to forget about other acute and chronic 359 diseases, such as HFs. This draws attention to the enhancement of health services' capacity 360 during emergencies, focusing on the prevention of collateral damage to patients with other 361 diseases, which should be an integral part of any preparedness and response plan aiming to tackle 362 health crises. 


\section{References}

364 1. Tedesco D, Gibertoni D, Rucci P, et al (2018) Impact of rehabilitation on mortality and

365 readmissions after surgery for hip fracture. BMC Health Serv Res 18:701.

366 https://doi.org/10.1186/s12913-018-3523-x

367 2. Svedbom A, Hernlund E, Ivergård M, et al (2013) Osteoporosis in the European Union:

368 A compendium of country-specific reports. Arch Osteoporos 8:. https://doi.org/10.1007/s11657-

369 013-0137-0

370 3. Sambrook P, Cooper C (2006) Osteoporosis. Lancet (London, England) 367:2010-8.

371 https://doi.org/10.1016/S0140-6736(06)68891-0

372 4. Beaupre LA, Khong H, Smith C, et al (2019) The impact of time to surgery after hip

373 fracture on mortality at 30- and 90-days: Does a single benchmark apply to all? Injury 50:950-

374 955. https://doi.org/10.1016/j.injury.2019.03.031

375 5. Haentjens P, Magaziner J, Colón-Emeric CS, et al (2010) Meta-analysis: excess mortality

376 after hip fracture among older women and men. Ann Intern Med 152:380-90.

377 https://doi.org/10.7326/0003-4819-152-6-201003160-00008

378 6. Hansen L, Mathiesen AS, Vestergaard P, et al (2013) A health economic analysis of

379 osteoporotic fractures: who carries the burden? Arch Osteoporos 8:126.

380 https://doi.org/10.1007/s11657-013-0126-3

381 7. Moja L, Piatti A, Pecoraro V, et al (2012) Timing Matters in Hip Fracture Surgery:

382 Patients Operated within 48 Hours Have Better Outcomes. A Meta-Analysis and Meta-

383 Regression of over 190,000 Patients. PLoS One 7:. https://doi.org/10.1371/journal.pone.0046175

384 8. Braithwaite RS, Col NF, Wong JB (2003) Estimating Hip Fracture Morbidity, Mortality

385 and Costs. J Am Geriatr Soc 51:364-370. https://doi.org/10.1046/j.1532-5415.2003.51110.x

386 9. Carretta E, Bochicchio V, Rucci P, et al (2011) Hip fracture: effectiveness of early

387 surgery to prevent 30-day mortality. Int Orthop 35:419-24. https://doi.org/10.1007/s00264-010-

388 1004-x

389 10. Shiga T, Wajima Z, Ohe Y (2008) Is operative delay associated with increased mortality

390 of hip fracture patients? Systematic review, meta-analysis, and meta-regression. Can J Anesth

391 55:146-154. https://doi.org/10.1007/BF03016088

392 11. National Institute for Health and Care Excellance (NICE) (2017) The management of hip

393 fracture in adults. Natl. Clin. Guidel. Cent. 1-628 
394 12. SIOT (2021) Linee Guida - Fratture del Femore Prossimale nell'Anziano

395 13. Bhandari M, Swiontkowski M (2017) Management of Acute Hip Fracture. N Engl J Med 396 377:2053-2062. https://doi.org/10.1056/NEJMcp1611090

397 14. Ministero della Salute - Agenas (2019) Programma Nazionale Esiti - PNE Edizione 2019

398 15. OECD/European Union (2020) Health at a Glance: Europe 2020. OECD

399 16. Gibertoni D, Adja KYC, Golinelli D, et al (2021) Patterns of COVID-19 related excess

400 mortality in the municipalities of Northern Italy during the first wave of the pandemic. Health

401 Place 67:102508. https://doi.org/10.1016/j.healthplace.2021.102508

402 17. Lavezzo E, Franchin E, Ciavarella C, et al (2020) Suppression of a SARS-CoV-2

403 outbreak in the Italian municipality of Vo'. Nature 584:425-429. https://doi.org/10.1038/s41586$404 \quad 020-2488-1$

405 18. Placella G, Salvato D, Delmastro E, et al (2020) CoViD-19 and ortho and trauma 406 surgery: The Italian experience. Injury 51:1403-1405

407 19. Regione Emilia - Romagna Direzione Generale Cura della Persona Salute e Welfare 408 (2020) Piano di riorganizzazione per i ricoveri in regime di terapia intensiva e in aree di 409 assistenza ad alta intensità di cura

410 20. Golinelli D, Toscano F, Bucci A, et al (2017) Health Expenditure and All-Cause 411 Mortality in the 'Galaxy' of Italian Regional Healthcare Systems: A 15-Year Panel Data 412 Analysis. Appl Health Econ Health Policy 15:773-783. https://doi.org/10.1007/s40258-017413 0342-x

414 21. Gatto M, Bertuzzo E, Mari L, et al (2020) Spread and dynamics of the COVID-19 415 epidemic in Italy: Effects of emergency containment measures. Proc Natl Acad Sci U S A 416 117:10484-10491. https://doi.org/10.1073/pnas.2004978117

417 22. Rivieccio BA, Luconi E, Boracchi P, et al (2020) Heterogeneity of covid-19 outbreak in 418 italy. Acta Biomed 91:31-34. https://doi.org/10.23750/abm.v91i2.9579

419 23. Williams GA, Maier CB, Scarpetti G, et al (2020) What strategies are countries using to 420 expand health workforce surge capacity during the Covid-19 pandemic? TEN 26:51

421 24. Lenzi J, Luciano L, McDonald KM, et al (2014) Empirical examination of the indicator 422 'pediatric gastroenteritis hospitalization rate' based on administrative hospital data in Italy. Ital J 423 Pediatr 40:14. https://doi.org/10.1186/1824-7288-40-14 
424 25. Quan H, Sundararajan V, Halfon P, et al (2005) Coding Algorithms for Defining

425 Comorbidities in ICD-9-CM and ICD-10 Administrative Data. Med Care 43:

426 26. Shadish WR, Cook TD, Campbell DT (2002) Quasi-experiments: interrupted time-series

427 designs. In: Experimental and quasi-experimental designs for generalized causal inference.

428 Houghton Mifflin Company, Boston, USA, pp 171-206

429 27. Linden A, Adams JL (2011) Applying a propensity score-based weighting model to 430 interrupted time series data: improving causal inference in programme evaluation. J Eval Clin 431 Pract 17:1231-8. https://doi.org/10.1111/j.1365-2753.2010.01504.x

432 28. Cumby RE, Huizinga J (1992) Testing the Autocorrelation Structure of Disturbances in 433 Ordinary Least Squares and Instrumental Variables Regressions. Econometrica 60:185-195. 434 https://doi.org/10.2307/2951684

435 29. Linden A (2015) Conducting Interrupted Time-series Analysis for Single- and Multiple436 group Comparisons. Stata J 15:480-500. https://doi.org/10.1177/1536867X1501500208 437 30. Fantini MP, Fabbri G, Laus M, et al (2011) Determinants of surgical delay for hip 438 fracture. Surgeon 9:130-134. https://doi.org/10.1016/j.surge.2010.11.031

439 31. Lizaur-Utrilla A, Gonzalez-Navarro B, Vizcaya-Moreno MF, et al (2019) Reasons for 440 delaying surgery following hip fractures and its impact on one year mortality. Int Orthop 441 43:441-448. https://doi.org/10.1007/s00264-018-3936-5

442 32. Sheehan KJ, Sobolev B, Villán Villán YF, Guy P (2017) Patient and system factors of 443 time to surgery after hip fracture: a scoping review. BMJ Open 7:e016939.

444 https://doi.org/10.1136/bmjopen-2017-016939

445 33. Casula M, Terlizzi A, Toth F (2020) I servizi sanitari regionali alla prova del COVID-19. 446 Riv Ital di Polit Pubbliche XV:307-336. https://doi.org/10.1483/98732

447 34. Italian Civil Protection Department (2020) Official Italian data about COVID-19. In: 448 Online. https://github.com/pcm-dpc/COVID-19. Accessed 28 Feb 2021

449 35. Andreata M, Faraldi M, Bucci E, et al (2020) Operating room efficiency and timing 450 during coronavirus disease 2019 outbreak in a referral orthopaedic hospital in Northern Italy. Int 451 Orthop 44:2499-2504. https://doi.org/10.1007/s00264-020-04772-X

452 36. Campo G, Fortuna D, Berti E, et al (2021) In- and out-of-hospital mortality for 453 myocardial infarction during the first wave of the COVID-19 pandemic in Emilia-Romagna, 
454 Italy: A population-based observational study. Lancet Reg Heal - Eur 100055.

455 https://doi.org/10.1016/j.lanepe.2021.100055

456 37. Rosenbaum L (2020) The Untold Toll — The Pandemic's Effects on Patients without

457 Covid-19. N Engl J Med 382:2368-2371. https://doi.org/10.1056/NEJMms2009984

458 38. Jeffery MM, D’Onofrio G, Paek H, et al (2020) Trends in Emergency Department Visits 459 and Hospital Admissions in Health Care Systems in 5 States in the First Months of the COVID46019 Pandemic in the US. JAMA Intern Med 180:1328.

461 https://doi.org/10.1001/jamainternmed.2020.3288

462 39. Santi L, Golinelli D, Tampieri A, et al (2020) Non-COVID-19 patients in times of 463 pandemic: Decreased emergency department visits and increased out-of-hospital mortality in 464 Northern Italy. medRxiv 2020.11.10.20229237

465 40. Dolci A, Marongiu G, Leinardi L, et al (2020) The Epidemiology of Fractures and 466 Muskulo-Skeletal Traumas During COVID-19 Lockdown: A Detailed Survey of 17.591 Patients 467 in a Wide Italian Metropolitan Area. Geriatr Orthop Surg Rehabil 11:

468 https://doi.org/10.1177/2151459320972673

469 41. Maniscalco P, Poggiali E, Quattrini F, et al (2020) Proximal femur fractures in covid-19 470 emergency: The experience of two orthopedics and traumatology departments in the first eight 471 weeks of the Italian epidemic. Acta Biomed 91:89-96. https://doi.org/10.23750/abm.v91i2.9636

472 42. Nuñez JH, Sallent A, Lakhani K, et al (2020) Impact of the COVID-19 Pandemic on an 473 Emergency Traumatology Service: Experience at a Tertiary Trauma Centre in Spain. Injury 474 51:1414-1418. https://doi.org/10.1016/j.injury.2020.05.016

475 43. Ogliari G, Lunt E, Ong T, et al (2020) The impact of lockdown during the COVID-19 476 pandemic on osteoporotic fragility fractures: an observational study. Arch Osteoporos 15:156. 477 https://doi.org/10.1007/s11657-020-00825-1

478 44. Grassi A, Pizza N, Tedesco D, Zaffagnini S (2020) The COVID-19 outbreak in Italy: 479 perspectives from an orthopaedic hospital. Int Orthop 44:1543-1547.

480 https://doi.org/10.1007/s00264-020-04617-7

481 45. Bärnighausen T, Tugwell P, Røttingen JA, et al (2017) Quasi-experimental study designs 482 series_-paper 4: uses and value. J Clin Epidemiol 89:21-29.

483 https://doi.org/10.1016/j.jclinepi.2017.03.012 


\section{TABLES}

485

486 Table 1 Hospital admissions for hip fracture $(\times 100,000$ inhabitants $)$ in Piedmont and Emilia-

487 Romagna, Italy, by 13-week observation period.

$488{ }^{a}$ By sex and age with direct standardization to Italy's 2020 elderly population ( $\geq 65$ years).

489

490 Table 2 Regression table of interrupted time-series analysis on weekly sex- and age-standardized

491 hip-fracture hospitalization rates in Piedmont and Emilia-Romagna before and after Italy's

492 COVID-19 national lockdown.

493 Notes: Data observed the year before (2018/19) are used for comparison. $T_{t}$ is time since the start of the 494 study (December 11), $X_{t}$ is an indicator variable that equals 1 in the weeks 11 to 23 of the tropical year 495 (March 11, 2020/March 12, 2019 to June 9, 2020/June 10, 2019), and $Z$ is an indicator variable that 496 equals 1 in the experimental time series (December 11, 2019 to June 9, 2020). The post-lockdown trend 497 between March 11, 2020 and June 9, 2020 can be obtained as $\beta\left(T_{t}\right)+\beta\left(Z T_{t}\right)+\beta\left(X_{t} T_{t}\right)+\beta\left(Z X_{t} T_{t}\right)$. 498 Abbreviations: $C I$, confidence interval.

499

500 Table 3 Regression table of interrupted time-series analysis on weekly sex-, age- and 501 comorbidity-standardized percentage of surgery for hip fracture in Piedmont and Emilia502 Romagna in the 13 weeks before and after Italy's COVID-19 national lockdown.

503 Notes: Data observed the year before (2018/19) are used for comparison. $T_{t}$ is time since the start of the 504 study (December 11), $X_{t}$ is an indicator variable that equals 1 in the weeks 11 to 23 of the tropical year 505 (March 11, 2020/March 12, 2019 to June 9, 2020/June 10, 2019), and $Z$ is an indicator variable that 506 equals 1 in the experimental time series (December 11, 2019 to June 9, 2020). The post-lockdown trend 507 between March 11, 2020 and June 9, 2020 can be obtained as $\beta\left(T_{t}\right)+\beta\left(Z T_{t}\right)+\beta\left(X_{t} T_{t}\right)+\beta\left(Z X_{t} T_{t}\right)$. 


\section{FIGURES}

509

510 Fig. 1 Interrupted time-series analysis of weekly sex- and age-standardized hip-fracture

511 hospitalization rates in Piedmont and Emilia-Romagna in the 13 weeks before and after Italy's

512 COVID-19 national lockdown (dashed vertical line).

513 Note: Data observed the year before (2018/19) are used for comparison. The last day of the control period

514 is June 10, 2019, because 2019 is a common (non-leap) year.

515

516 Fig. 2 Hip-fracture surgery initiated within 2 days, within 1 day and on the same day as hospital

517 admission in Piedmont and Emilia-Romagna, Italy, by 13-week observation period.

518 Note: All the rates are standardized by sex, age and enhanced Charlson index with direct standardization

519 to the overall composition of hip fractures included in the study.

520

521 Fig. 3 Interrupted time-series analysis of weekly sex-, age- and comorbidity-standardized

522 percentages of hip-fracture surgery in Piedmont and Emilia-Romagna in the 13 weeks before and 523 after Italy's COVID-19 national lockdown (dashed vertical line).

524 Note: Data observed the year before (2018/19) are used for comparison. The last day of the control period 525 is June 10, 2019, because 2019 is a common (non-leap) year. 


\section{SUPPLEMENTS}

527

528 Fig. S1 Prevalence of COVID-19-associated hospitalizations and cumulative incidence of

529 COVID-19 cases $(\times 100,000$ population $)$ in Piedmont and Emilia-Romagna and Italy between

530 February 24, 2020 and June 9, 2020.

531 Note: Dashed vertical line is Italy's COVID-19 national lockdown (March 10, 2020).

532 Source: Dipartimento della protezione civile.

533

534 Table S1 Characteristics of hip-fracture patients and admitting hospitals in Piedmont and Emilia-

535 Romagna between November 20, 2019 and June 30, 2020, by 13-week period; values are counts 536 (percentages).

537

538 Table S2 Hip-fracture surgery (\%) initiated within 2 days, within 1 day and on the same day as 539 hospital admission in Piedmont and Emilia-Romagna, Italy, by 13-week observation period; 540 secondary diagnoses of upper femur fracture are excluded (153 in Piedmont and 125 in Emilia541 Romagna).

542 a By sex, age and enhanced Charlson index with direct standardization to the overall composition of hip 543 fractures included in the study.

544

545 Fig. S2 Interrupted time-series analysis of weekly sex-, age- and comorbidity-standardized 546 percentages of hip-fracture surgery in Piedmont and Emilia-Romagna in the 13 weeks before and 547 after Italy's COVID-19 national lockdown (dashed vertical line); secondary diagnoses of upper 548 femur fracture are excluded (153 in Piedmont and 125 in Emilia-Romagna).

549 Note: Data observed the year before (2018/19) are used for comparison. The last day of the control period 550 is June 10, 2019, because 2019 is a common (non-leap) year. 
Table $\mathbf{1}$ (on next page)

Tables 


\section{TABLES}

2

3 Table 1 Hospital admissions for hip fracture $(\times 100,000$ inhabitants $)$ in Piedmont and Emilia-

4 Romagna, Italy, by 13-week observation period.

\begin{tabular}{lcccccc}
\hline \multirow{2}{*}{$\begin{array}{l}\text { Thirteen-week observation } \\
\text { period }\end{array}$} & $n$ & $\begin{array}{c}\text { Crude } \\
\text { rate }\end{array}$ & $\begin{array}{c}\text { Standardized } \\
\text { rate }^{\text {a }}\end{array}$ & $n$ & $\begin{array}{c}\text { Emilia-Romagna } \\
\text { Crude } \\
\text { rate }\end{array}$ & $\begin{array}{c}\text { Standardized } \\
\text { rate }^{\text {a }}\end{array}$ \\
\hline Dec-11-2018 to Mar-11-2019 & 1788 & 161.4 & 159.5 & 1720 & 160.5 & 153.3 \\
Mar-12-2019 to Jun-10-2019 & 1687 & 152.3 & 150.8 & 1731 & 161.6 & 154.6 \\
Dec-11-2019 to Mar-10-2020 & 1698 & 152.2 & 148.8 & 1730 & 160.3 & 152.2 \\
Mar-11-2020 to Jun-09-2020 & 1388 & 124.4 & 121.6 & 1465 & 135.7 & 128.7 \\
\hline
\end{tabular}

$5 \quad$ a By sex and age with direct standardization to Italy's 2020 elderly population ( $\geq 65$ years). 
6 Table 2 Regression table of interrupted time-series analysis on weekly sex- and age-standardized 7 hip-fracture hospitalization rates in Piedmont and Emilia-Romagna before and after Italy's 8 COVID-19 national lockdown.

\begin{tabular}{lcccc}
\hline \multirow{2}{*}{ Variable } & \multicolumn{2}{c}{ Piedmont } & \multicolumn{2}{c}{ Emilia-Romagna } \\
\cline { 2 - 5 } & Coefficient $(95 \% \mathrm{CI})$ & $P$-value & Coefficient $(95 \% \mathrm{CI})$ & $P$-value \\
\hline Intercept & $12.59(11.07,14.12)$ & $<0.001$ & $12.99(12.12,13.87)$ & $<0.001$ \\
$T_{t}$ & $-0.06(-0.24,0.13)$ & 0.546 & $-0.17(-0.29,-0.04)$ & 0.010 \\
$X_{t}$ & $-0.71(-2.34,0.91)$ & 0.379 & $-0.61(-2.46,1.24)$ & 0.510 \\
$X_{t} T_{t}$ & $0.12(-0.14,0.38)$ & 0.353 & $0.37(0.15,0.58)$ & 0.001 \\
$Z$ & $-0.55(-2.73,1.64)$ & 0.616 & $0.04(-1.08,1.15)$ & 0.949 \\
$Z T_{t}$ & $-0.04(-0.32,0.24)$ & 0.770 & $-0.04(-0.19,0.12)$ & 0.627 \\
$Z X_{t}$ & $-2.22(-4.88,0.44)$ & 0.100 & $-1.52(-3.69,0.65)$ & 0.164 \\
$Z X_{t} T_{t}$ & $0.21(-0.18,0.60)$ & 0.291 & $0.06(-0.20,0.32)$ & 0.629 \\
\hline
\end{tabular}

9 Notes: Data observed the year before (2018/19) are used for comparison. $T_{t}$ is time since the start of the study (December 11), $X_{t}$ is an indicator variable that equals 1 in the weeks 11 to 23 of the tropical year

11 (March 11, 2020/March 12, 2019 to June 9, 2020/June 10, 2019), and $Z$ is an indicator variable that

12 equals 1 in the experimental time series (December 11, 2019 to June 9, 2020). The post-lockdown trend 13 between March 11, 2020 and June 9, 2020 can be obtained as $\beta\left(T_{t}\right)+\beta\left(Z T_{t}\right)+\beta\left(X_{t} T_{t}\right)+\beta\left(Z X_{t} T_{t}\right)$. Abbreviations: $C I$, confidence interval. 
15 Table 3 Regression table of interrupted time-series analysis on weekly sex-, age- and

Piedmont

Coefficient $(95 \% \mathrm{CI}) \quad P$-value $\quad$ Coefficient $(95 \% \mathrm{CI}) \quad P$-value

18

19

20

21

22

23

Variable
Surgery within 2 days

Intercept

$T_{t}$

$X_{t}$

$X_{t} T_{t}$

$Z$

$Z T_{t}$

$Z X_{t}$

$Z X_{t} T_{t}$

Surgery within 1 day

Intercept

$T_{t}$

$X_{t}$

$X_{t} T_{t}$

$Z$

$Z T_{t}$

$Z X_{t}$

$Z X_{t} T_{t}$

Surgery on the same day

Intercept

$T_{t}$

$X_{t}$

$X_{t} T_{t}$

$Z$

$Z T_{t}$

$Z X_{t}$

$Z X_{t} T_{t}$

\begin{tabular}{cccc}
$72.36(64.95,79.77)$ & $<0.001$ & $72.73(65.55,79.91)$ & $<0.001$ \\
$-0.59(-1.58,0.40)$ & 0.238 & $0.59(-0.25,1.43)$ & 0.166 \\
$1.37(-4.99,7.72)$ & 0.667 & $-4.39(-11.21,2.42)$ & 0.201 \\
$0.80(-0.25,1.86)$ & 0.133 & $-0.78(-1.80,0.25)$ & 0.133 \\
$-6.84(-16.14,2.45)$ & 0.145 & $-3.11(-14.56,8.34)$ & 0.587 \\
$1.10(-0.35,2.56)$ & 0.133 & $0.69(-0.71,2.09)$ & 0.328 \\
$-8.53(-21.31,4.26)$ & 0.186 & $-4.56(-13.73,4.60)$ & 0.321 \\
$-1.04(-2.75,0.67)$ & 0.227 & $-1.79(-3.37,-0.22)$ & 0.027 \\
& & & \\
$46.62(38.34,54.90)$ & $<0.001$ & $42.23(35.33,49.13)$ & $<0.001$ \\
$-0.30(-1.35,0.74)$ & 0.559 & $-0.08(-0.93,0.78)$ & 0.858 \\
$-2.49(-8.94,3.96)$ & 0.441 & $0.57(-8.19,9.33)$ & 0.897 \\
$0.68(-0.42,1.77)$ & 0.220 & $0.15(-1.02,1.32)$ & 0.795 \\
$-6.24(-16.29,3.80)$ & 0.217 & $-5.50(-14.36,3.35)$ & 0.217 \\
$0.95(-0.49,2.39)$ & 0.192 & $1.69(0.58,2.80)$ & 0.004 \\
$0.28(-15.01,15.57)$ & 0.971 & $0.66(-11.20,12.53)$ & 0.911 \\
$-1.40(-3.34,0.53)$ & 0.151 & $-4.04(-5.59,-2.49)$ & $<0.001$ \\
& & & \\
$15.77(11.47,20.08)$ & $<0.001$ & $10.41(6.73,14.10)$ & $<0.001$ \\
$-0.51(-1.03,0.01)$ & 0.053 & $-0.34(-0.85,0.17)$ & 0.181 \\
$2.99(0.01,5.98)$ & 0.050 & $1.27(-3.22,5.75)$ & 0.572 \\
$0.61(0.05,1.17)$ & 0.032 & $0.50(-0.08,1.08)$ & 0.088 \\
$-4.08(-9.17,1.01)$ & 0.113 & $-3.62(-8.56,1.33)$ & 0.147 \\
$0.64(0.01,1.27)$ & 0.048 & $0.93(0.19,1.67)$ & 0.015 \\
$-5.80(-11.37,-0.22)$ & 0.042 & $0.30(-7.51,8.11)$ & 0.938 \\
$-0.57(-1.37,0.22)$ & 0.154 & $-2.15(-3.15,-1.16)$ & $<0.001$ \\
\hline
\end{tabular}

Notes: Data observed the year before (2018/19) are used for comparison. $T_{t}$ is time since the start of the study (December 11), $X_{t}$ is an indicator variable that equals 1 in the weeks 11 to 23 of the tropical year (March 11, 2020/March 12, 2019 to June 9, 2020/June 10, 2019), and $Z$ is an indicator variable that equals 1 in the experimental time series (December 11, 2019 to June 9, 2020). The post-lockdown trend between March 11, 2020 and June 9, 2020 can be obtained as $\beta\left(T_{t}\right)+\beta\left(Z T_{t}\right)+\beta\left(X_{t} T_{t}\right)+\beta\left(Z X_{t} T_{t}\right)$. 
Figure 1

Interrupted time-series analysis of weekly sex- and age-standardized hip-fracture hospitalization rates in Piedmont and Emilia-Romagna in the 13 weeks before and after Italy's COVID-19 national lockdown (dashed vertical line).

Notes: Data observed the year before (2018/19) are used for comparison. The last day of the control period is June 10, 2019, because 2019 is a common (non-leap) year. 


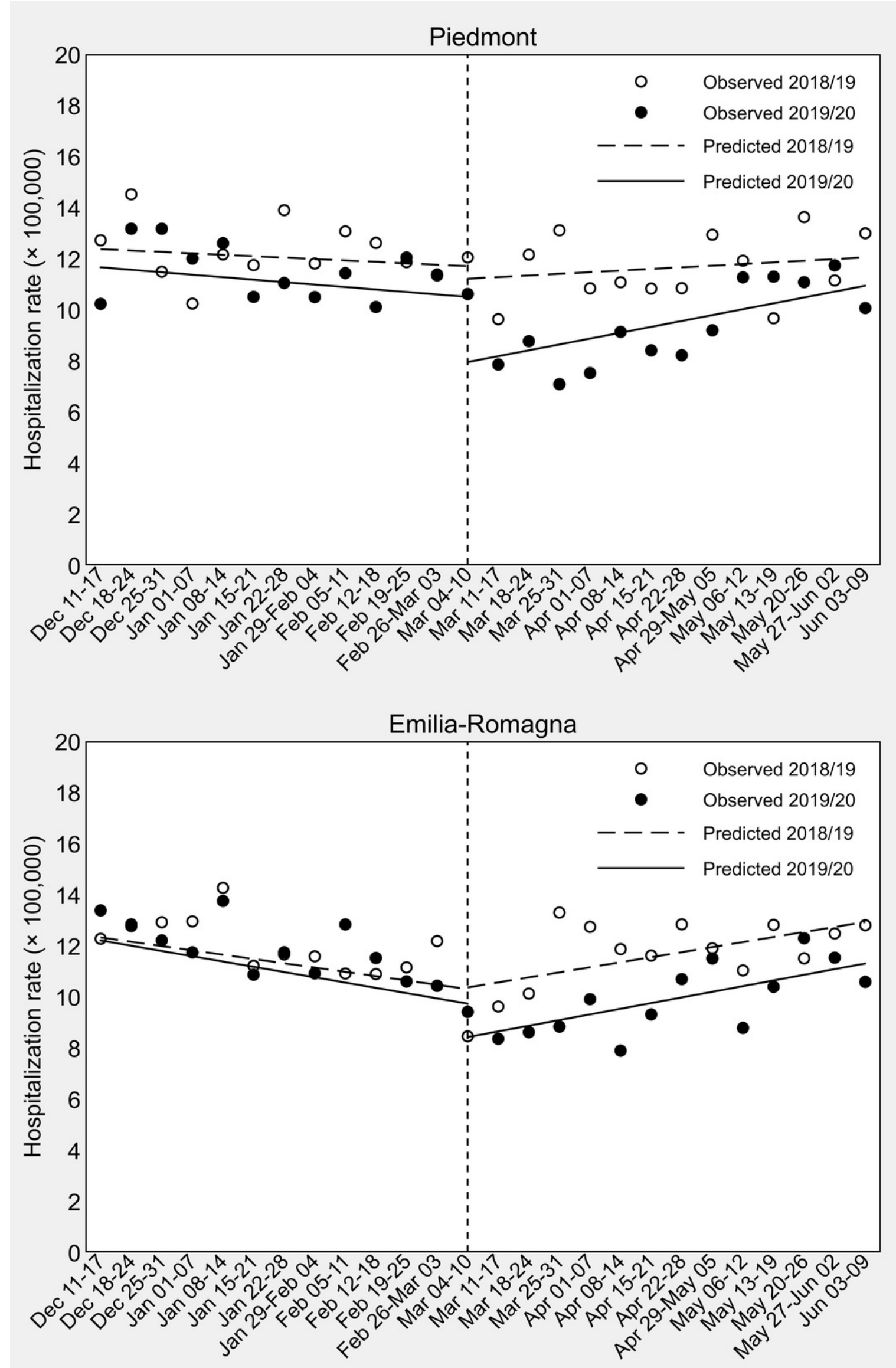




\section{Figure 2}

Hip-fracture surgery initiated within 2 days, within 1 day and on the same day as hospital admission in Piedmont and Emilia-Romagna, Italy, by 13-week observation period.

Note: All the rates are standardized by sex, age and enhanced Charlson index with direct standardization to the overall composition of hip fractures included in the study.

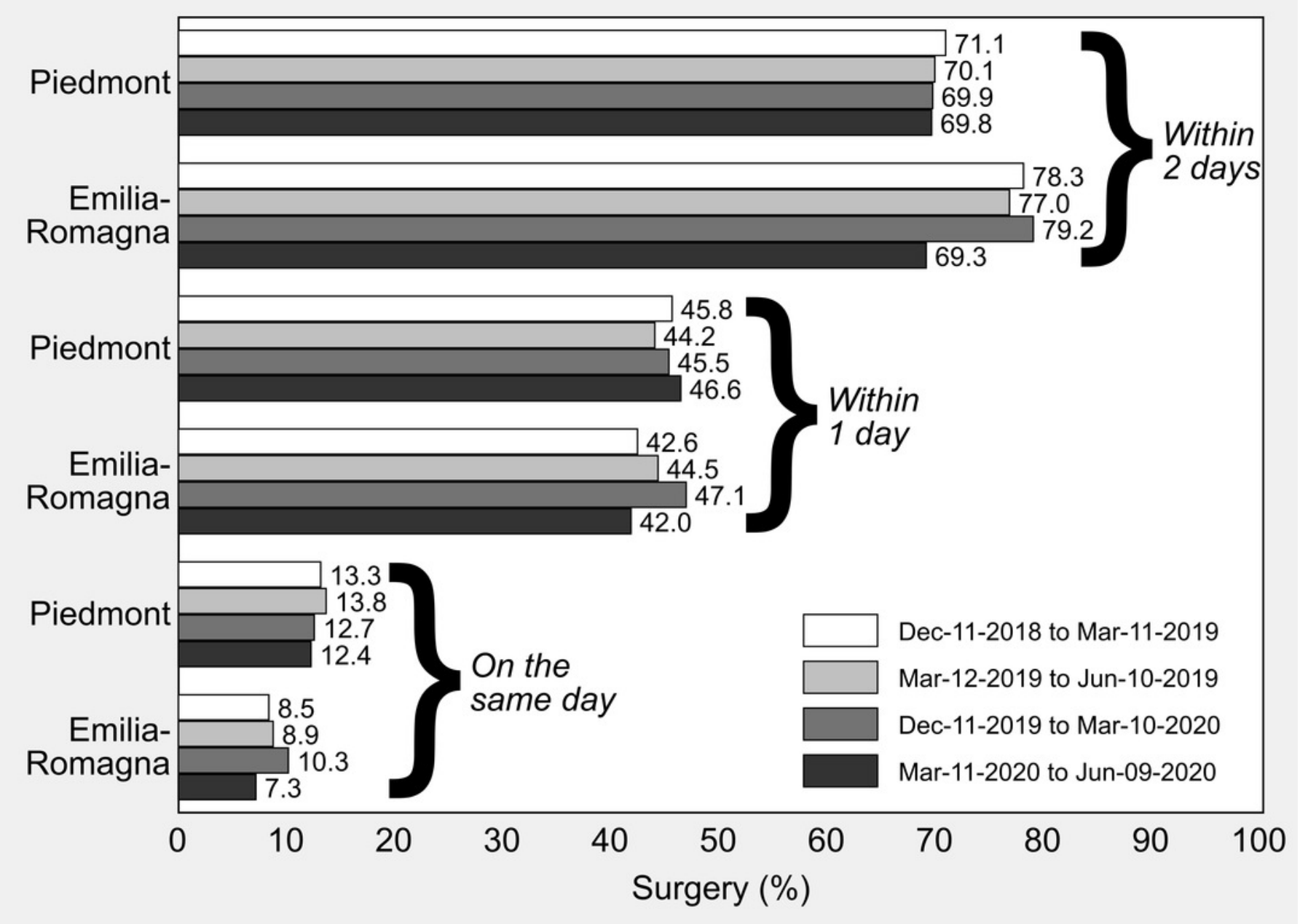




\section{Figure 3}

Interrupted time-series analysis of weekly sex-, age- and comorbidity-standardized percentages of hip-fracture surgery in Piedmont and Emilia-Romagna in the 13 weeks before and after Italy's COVID-19 national lockdown (dashed vertical line).

Note: Data observed the year before (2018/19) are used for comparison. The last day of the control period is June 10, 2019, because 2019 is a common (non-leap) year. 


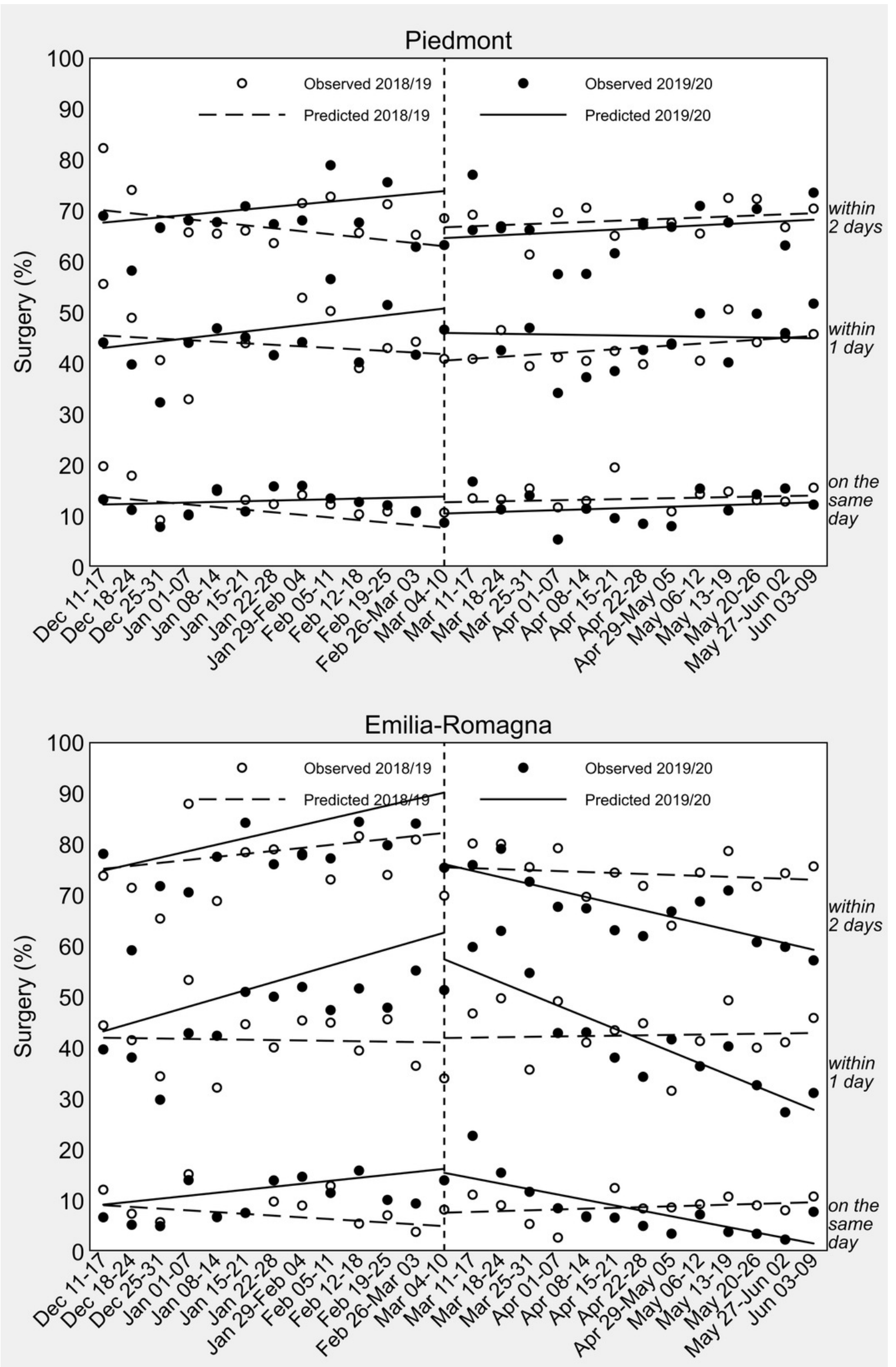

